\title{
Publicidad política y su incidencia en la participación de usuarios en redes sociales: elecciones presidenciales de Colombia, 2018
}

\author{
Carlos Fernando Osorio Andrade ${ }^{1}$ id \\ Edwin Arango Espinal ${ }^{2}$ \\ Hector Augusto Rodriguez Orejuela ${ }^{3}$ (i)
}

\begin{abstract}
La investigación presentada en este artículo analiza las estrategias de los anuncios políticos publicados en Twitter por los candidatos a la segunda vuelta de la elección presidencial de Colombia, en el año 2018. Se identificó el propósito y el enfoque del mensaje de 425 posts y se aplicó un modelo de regresión binomial negativa, para establecer los efectos de estos elementos sobre la participación de los usuarios en Twitter medida por likes, comentarios y retweets. Hubo dos hallazgos relevantes: a) la publicidad política agresiva - cuando los candidatos atacan, o se defienden de un oponente - aumenta la participación de los usuarios en Twitter; b) los mensajes con temas personales del candidato tienen mayor respaldo social que las publicaciones que abordan propuestas políticas.
\end{abstract}

Palabras clave: publicidad política; Twitter; emociones

\section{Introducción}

La publicidad es una de las herramientas más importantes de la estrategia del marketing político; particularmente, por la capacidad que tiene para influir sobre el comportamiento de los votantes (Kaid, 2001). Con el advenimiento de internet y las redes sociales, la publicidad política se adapta a los ecosistemas electrónicos y empieza a jugar un papel importante en los procesos electorales de todo el mundo (Sohal y Kaur, 2018).

Esta relevancia de los anuncios políticos -en el contexto de las redes sociales como Facebook, YouTube y Twitter - no ha pasado inadvertida por la academia (Borah, 2016; Williams y Gulati, 2017). Cada vez más, los científicos sociales se preocupan por explorar la influencia de la publicidad política sobre las actitudes y comportamientos del electorado.

\footnotetext{
${ }^{1}$ Universidad del Valle. Cali (Valle del Cauca), Colombia.

E-mail: <carlos.fernando.osorio@correounivalle.edu.co>.

2 Universidad del Valle. Caicedonia (Valle del Cauca), Colombia.

E-mail: <edwin.arango@correounivalle.edu.co>.

3 Universidad del Valle. Cali (Valle del Cauca), Colombia.

E-mail: <hector.augusto.rodriguez@correounivalle.edu.co>.
} 
No obstante, aún no existe un cuerpo del conocimiento suficientemente amplio, para dar cuenta de los efectos que ejercen los anuncios políticos electrónicos sobre los votantes (Shah et al., 2007).

De hecho, la influencia de la publicidad política sobre el respaldo social, conocido también como la participación de los usuarios en redes sociales, ha sido estudiada de manera escasa, lo que implica que la comprensión de sus efectos sigue siendo incompleta (Borah, 2016; Spenkuch y Toniatti, 2018). Aunque existen estudios exploratorios que caracterizan las publicaciones políticas en redes sociales (Shah et al., 2007; Herrero y Benoit, 2009; Williams y Gulati, 2017), no se encontraron, hasta el momento, documentos que establezcan relaciones de causalidad entre la publicidad política digital y la participación de los votantes en redes sociales.

El propósito de este artículo consiste en comprender, de una manera más clara y precisa, la influencia que ejercen las estrategias de publicidad política -utilizadas en publicaciones de redes sociales- sobre el respaldo de los usuarios. Se exploró el papel de Twitter como herramienta de promoción política, durante la segunda vuelta de la campaña presidencial de Colombia de 2018. Concretamente, se analizaron las publicaciones realizadas por Iván Duque y Gustavo Petro, en el periodo comprendido entre el 27 de mayo y el 27 de junio de 2018.

Para identificar y operacionalizar las características de la publicidad política en publicaciones de Twitter, se empleó la teoría del análisis funcional, en la investigación de los debates electorales, propuesta por Benoit. Específicamente, se identificaron el propósito y el enfoque del mensaje como elementos relevantes de los anuncios políticos en redes sociales (Benoit, 2000, 2001; Borah, 2016).

El respaldo social de los usuarios en Twitter se operacionalizó mediante likes, retweets y comentarios; estas métricas representan el rendimiento de las publicaciones, de acuerdo con la interacción generada. Después, se formularon preguntas de investigación para establecer los efectos de las características de las publicaciones sobre la participación digital de los ciudadanos. Para responder dichas preguntas, se analizaron 425 post de Twitter, correspondientes al total de tweets difundidos por Iván Duque y Gustavo Petro, durante la segunda vuelta presidencial de Colombia en 2018 y se aplicó un modelo de regresión binomial negativa, para explorar los efectos de las características de los anuncios sobre el respaldo social de los usuarios.

Los resultados obtenidos ofrecen modelos significativos que sugieren relaciones importantes entre las características de los anuncios y la participación de los ciudadanos en Twitter; también, muestran tres líneas de contribución a la literatura académica. En primer lugar, el empleo de la teoría del análisis funcional de Benoit, para caracterizar los mensajes políticos en un contexto cultural y económico diferente a los abordados por estudios previos. En segundo lugar, el modelo de la teoría del análisis funcional, que se probó, de manera empírica. Hasta el momento, solamente existen estudios que clasifican las publicaciones de los candidatos de acuerdo con la teoría del análisis funcional. No se 
encontraron estudios empíricos que establezcan los efectos de las categorías propuestas por dicha teoría, en función de la participación de usuarios en redes sociales. Finalmente, en tercer lugar, los hallazgos de esta investigación contribuyen al debate de la efectividad de la publicidad política negativa; los resultados indican que este tipo de propaganda mejora la participación de los electores colombianos en redes sociales.

Este artículo se divide en cinco apartados incluida la presente "Introducción". En el segundo apartado, el marco teórico, con "Publicidad política y su influencia sobre el electorado", "Internet, redes sociales y publicidad política 2.0" y "Teoría del análisis funcional para la investigación de los debates electorales", se abordan el contexto teórico donde se plantea el papel de internet y las redes sociales sobre la publicidad política 2.0 También, se presenta la teoría del análisis funcional para la investigación de los debates electorales. En el tercer apartado, "Metodología", se explica la metodología llevada a cabo para cumplir el objetivo de investigación. En el cuarto apartado se exponen los "Resultados" y hallazgos más importantes; finalmente, en el quinto apartado, se presentan las "Conclusiones" generales del texto.

\section{Publicidad política y su influencia sobre el electorado}

La literatura científica considera que la publicidad política es el elemento más importante en la estrategia del marketing político (Chaffee y Choe, 1980; Van Steenburg, 2015), y la define como el conjunto de mensajes controlados y difundidos, a través de cualquier medio de comunicación, con el propósito de impulsar intereses políticos de individuos, partidos, gobiernos u otro tipo de organizaciones ante la sociedad (Kaid y Bacha, 2006). Ciertos grupos de autores sostienen que los anuncios políticos podrían no solo influenciar la decisión de voto, sino manipular al electorado (Van Steenburg, 2015).

Durante décadas, los estudios académicos que analizan el papel de la publicidad política han reconocido efectos significativos de este tipo de promoción electoral sobre el comportamiento de las personas en elecciones populares (Evarts y Guido, 1974; Soley y Reid, 1982; Brader, 2005). No obstante, la mayoría de autores señala que aún no existe un cuerpo de conocimiento suficiente para dar cuenta precisa de la influencia que ejercen los anuncios sobre el electorado (Shah et al., 2007; Rao, Lee y Kim, 2009).

En líneas generales, la investigación de la publicidad política ha encontrado que los anuncios pueden ejercer efectos sobre las decisiones de voto de las personas, así como en la activación y el refuerzo de las opiniones (Berelson, Lazarsfeld y McPhee, 1954). Según Benoit (2000), el uso de publicidad política juega un rol trascendental en la construcción y el fortalecimiento de las relaciones entre los candidatos, el partido político y los votantes. Algunos estudios han comprobado que la publicidad política a menudo es utilizada por candidatos, con el objetivo de conducir a los votantes a centrarse en problemas y situaciones favorables para ellos mismos y desfavorables para sus oponentes (Abbe et al., 2003; Valentino, Hutchings y Williams, 2004). 
La investigación sobre los efectos de la publicidad política en el comportamiento de los electores también sugiere que los anuncios pueden ejercer persuasión sobre los votantes, aunque con efectos de corta duración (Franz y Ridout, 2007). Particularmente, los anuncios políticos parecen ser efectivos con electores poco informados e indecisos; pero no generan la misma influencia sobre votantes que tienen alto nivel de información sobre los candidatos (Ridout, Franz y Fowler, 2015, como se citó en Ridout, et al., 2017). En línea con estos planteamientos, Larreguy, Snyder y Marshall (2018) sugieren que la publicidad política solamente tiene influencia considerable en democracias poco maduras o inestables, mientras que presenta efectos insignificantes en democracias sólidas y organizadas.

Otras investigaciones han reconocido que la publicidad política tiene la capacidad de mejorar la confianza de los votantes, con la intención de recabar pensamientos e ideas por parte de individuos u organizaciones involucradas en política (Pathak y Kumar, 2012, como se citó en Seerat y Hansandaldeep, 2018). De hecho, los estrategas de campañas electorales creen firmemente que los anuncios pueden tener impacto en persuadir y movilizar a los ciudadanos (Williams y Gulati, 2017).

\section{Internet, redes sociales y publicidad política $\mathbf{2 . 0}$}

Con la llegada de internet y la transformación de los medios de comunicación (Flew, 2007), la vida cotidiana y las formas de comunicación han cambiado de manera notable (Harris y Sanborn, 2009). Esta revolución digital también ha representado un cambio en la manera de hacer publicidad política (Larson y Denton, 2014). Actualmente, los ciudadanos pasan, cada vez, menos tiempo frente a la televisión, pero dedican mucho a seguir campañas electorales a través de los medios digitales (Kaid y Postelnicu, 2005). De esta forma, las campañas políticas han tomado tintes bidireccionales, en las que los ciudadanos "de a pie" comienzan a jugar un rol más activo dentro del proceso electoral (Dimmick, Chen y Li, 2004).

Debido a su importancia en contiendas electorales, las redes sociales, cada vez, generan mayor interés en tácticas políticas; por tanto, la inversión -en estos medios digitales, como Facebook, YouTube y Twitter- ha crecido de manera sostenida en los últimos años (Mangold y Faulds, 2009). Para Smith (2013), seis de cada diez adultos utilizan las redes sociales, y dos tercios de estas personas son políticamente activas a través de medios electrónicos. Uno de los casos que más se cita en la literatura, para indicar la importancia de las redes sociales en procesos electorales, es el uso de redes, por parte de Obama, en las elecciones de 2008. Esto demostró que un candidato puede llegar con éxito a la audiencia y muy especialmente a los jóvenes (Dalton, 2015).

Aunque existen estudios exploratorios que caracterizan las tipologías de mensajes políticos en redes sociales (Borah, 2016; Sohal y Kaur, 2018), hacen falta investigaciones que prueben empíricamente los efectos que estas características pueden tener en la 
participación de usuarios. Bajo esta premisa, para futuros estudios sobre publicidad política, es pertinente tener en cuenta lo que Van Steenburg (2015) plantea: es necesario comprender el uso de tecnología en campañas electorales, respondiendo los siguientes interrogantes: ¿cómo están utilizando los políticos las redes sociales para conectarse con los votantes? y, ¿qué tipo de contenido recibe el mayor respaldo social en estas redes?

Teniendo en cuenta que no se encontraron, hasta el momento, estudios empíricos que prueben la influencia de las características de la publicidad política en redes sociales, sobre el respaldo social de los usuarios, a continuación, se presenta el marco conceptual sobre el cual se trabajó este artículo. Se plantea que el propósito y el enfoque del mensaje, elementos extraídos de la teoría del análisis funcional de Benoit, están relacionados con la participación de los usuarios en twitter mediante likes, retweets y comentarios.

\section{Teoría del análisis funcional para la investigación de los debates electorales}

La teoría del análisis funcional para la investigación de los debates electorales se ha consolidado como un modelo recurrente para analizar las estrategias de publicidad política en medios tradicionales, como la radio, la televisión y la prensa (Borah, 2016). Esta teoría sostiene que los candidatos utilizan tres estrategias para comunicar sus mensajes: anuncios de aclamación donde manifiestan sus propias fortalezas, anuncios de ataque (críticas a las debilidades de los oponentes) y anuncios de defensa cuando necesitan refutar el ataque de la competencia (Herrero y Benoit, 2009). Durante más de dos décadas, este modelo ha sido utilizado para estudiar cada debate presidencial de las elecciones estadounidenses, y aplicado en contextos como Israel (Benoit y Sheafer, 2006), Ucrania (Benoit y Klyukovski, 2006), Taiwan (Benoit, Wen y Yu, 2007) y Corea del Sur (Lee y Benoit, 2005).

\section{Propósito del mensaje}

Siguiendo la clasificación de los mensajes políticos que contempla la teoría funcional para el análisis de los debates electorales propuesta por Benoit (2000), se puede considerar a los anuncios que tienen por propósito la aclamación, como positivos, mientras que, la publicidad que apela al ataque, la defensa y la comparación entre candidatos, puede ser catalogada como negativa (Goldstein y Freedman, 2002). Existen varios documentos académicos que se han encargado de establecer los efectos de la publicidad política negativa sobre el comportamiento del electorado, encontrándose un debate importante en la literatura.

Inicialmente, Ansolabehere y Iyengar (1994) y Ansolabehere et al. (1994) proponen la hipótesis de la llamada "desmovilización", la cual sugiere que los anuncios políticos negativos (generalmente de ataque entre candidatos) desaniman a las personas a votar. Esta disminución en la participación de los votantes puede explicarse por varias 
razones, una de ellas es que los electores suelen hacerse más críticos, no solo con el candidato que es objetivo del ataque, sino también con quien difunde el mensaje negativo (Basil, Schooler y Reeves, 1991); por otra parte, aunque la publicidad negativa efectivamente afecta la opinión hacía el candidato que recibe el ataque, no quiere decir que mejore las actitudes de los votantes hacía el atacante, lo que genera un sentimiento generalizado de aversión ante la contienda electoral (Ansolabehere y Iyengar, 1994). También se ha hablado en la literatura del efecto boomerang, el cual indica que la publicidad negativa genera un rebote, es decir, genera un sentimiento de repulsión hacia el atacante y no hacía la victima (Merritt, 1984; Garramone, 1985; Borba, 2010), lo que se relaciona con el síndrome de la víctima, que básicamente implica que los votantes perciben a la publicidad agresiva como injusta, exagerada y deshonesta, lo que genera emociones positivas hacía el blanco del ataque, mejorando su imagen y aumentando la intención de voto ante este candidato (Garramone, 1985; Borba, 2010). En esta misma línea, Pinkleton, Um y Austin (2002) sostienen que la comunidad general encuentra menos útil a la publicidad política negativa para tomar decisiones de voto y Shah et al. (2007) sugieren que, si bien la publicidad política aumenta la intención de buscar información sobre los procesos electorales por parte de los votantes, los anuncios agresivos o de "ataque", por el contrario, disminuyen las motivaciones de buscar información a través de medios impresos y de difusión, al mismo tiempo que reducen la participación en la vida cívica y política.

A pesar de lo anterior, y como ya se ha mencionado, un número importante de investigaciones contradicen a esta corriente, señalando que la publicidad política negativa o los mensajes de campaña agresivos, en lugar de reducir el tamaño del electorado, estimulan la participación de los ciudadanos atrayendo nuevos votantes potenciales, en lo que se conoce como hipótesis de la estimulación. De hecho, estas investigaciones sugieren que la publicidad política negativa es importante para el sistema político, pues genera más información, establece un contraste entre las opciones electorales, aborda temas de interés para la sociedad y forma parte de la responsabilidad política (Finkle y Geer, 1998; Wattenberg y Brians, 1999; Goldstein y Freedman, 2002; Borba, 2010).

Teniendo en cuenta el debate alrededor de los efectos que generan los distintos propósitos de la publicidad política y frente a las pocas investigaciones que analizan este tema en el contexto de redes sociales como twitter, se describe la siguiente pregunta de investigación:

P1. ¿Cuál de los propósitos en publicidad política tiene mayor impacto en el respaldo social de los usuarios de redes sociales?

\section{Enfoque del mensaje}

La teoría del análisis funcional también asegura que cada publicidad política se sustenta en dos enfoques o cuestiones: a) las relacionadas con situaciones políticas; y b) 
aquellas que tienen que ver con aspectos personales del candidato (Herrero y Benoit, 2009; Borah, 2016). El primero se refiere a aquellos asuntos que conciernen al Estado, así como las propuestas para solucionar inconvenientes; es decir, a las promesas de campaña. El segundo, a las cuestiones relacionadas con el candidato: cuando el político habla de aptitudes o cualificaciones personales (Herrero y Benoit, 2009), por ejemplo, cuando el candidato presenta a su familia, sus intereses religiosos o al momento de hablar sobre su equipo de fútbol favorito. Aunque el enfoque del mensaje se ha tratado en varios documentos que utilizan la teoría del análisis funcional (Lee y Benoit, 2005; Benoit y Sheafer, 2006; Benoit y Klyukovski, 2006; Benoit, Wen y Yu, 2007), hasta el momento no se han encontrado investigaciones que establezcan los efectos de los tipos de enfoques sobre el comportamiento de los electores, y menos aún, sobre el comportamiento digital de los votantes; dado lo anterior se plantea la siguiente pregunta de investigación:

P2. ¿Cuál enfoque de la publicidad política tiene mejores resultados en la participación de usuarios en redes sociales?

\section{Metodología}

\section{Descripción de la muestra}

Se escogieron, para su revisión, 425 publicaciones de Twitter que corresponden al total de mensajes difundidos por Iván Duque y Gustavo Petro, en la segunda vuelta presidencial de Colombia, en 2018. Las piezas fueron extraídas a través de minería de datos -software Rstudio-, con las métricas de interés (likes, retweets y comentarios).

\section{Análisis de contenido}

Las publicaciones políticas realizadas por estos dos candidatos a la presidencia de Colombia fueron revisadas mediante análisis de contenido, utilizando la teoría del análisis funcional para la investigación de los debates electorales, propuesta por Benoit. Específicamente, se identificaron dos aspectos fundamentales en dichos mensajes: el propósito y el enfoque. El análisis de contenido es un método de investigación utilizado en mercadeo y ciencia política, para identificar las características de los anuncios políticos en medios tradicionales y digitales (De Vries, Gensler y Leeflang, 2012; Tafesse y Korneliussen, 2012; Borah, 2016; Sohal y Kaur, 2018).

Para este artículo, se emplearon dos personas que, previamente entrenadas para codificar, caracterizaron las piezas de comunicación de acuerdo con las tipologías propuestas, siguiendo la teoría del análisis funcional. Además de las categorías propuestas por dicha teoría, al revisar las piezas de comunicación, se incluyeron dos factores adicionales: comparación entre candidatos y campaña promocional. El primer factor se incluyó en la variable propósito del mensaje, que hace referencia a aquellas piezas de 
comunicación, en las que los candidatos se comparan con sus oponentes a través de imágenes de contraste o argumentos; esta categoría también fue utilizada por el estudio descriptivo de Borah (2016). El segundo factor hace referencia a aquellas piezas de comunicación cuyo solo propósito es hacer un llamado a la participación ciudadana, sin esgrimir argumento alguno. Por ejemplo, las canciones o jingles de campaña, así como las imágenes del tarjetón electoral que invitan a votar por el candidato (Tabla 1).

Tabla 1

Operacionalización de las variables

\begin{tabular}{|c|c|c|}
\hline Variable & Operacionalización & Detalles \\
\hline \multirow{4}{*}{$\begin{array}{l}\text { Propósito del } \\
\text { mensaje }\end{array}$} & $\begin{array}{c}\text { Aclamar al candidato } \\
\qquad(0-1)\end{array}$ & $\begin{array}{l}\text { Publicaciones positivas, en las que el candidato habla de } \\
\text { sus fortalezas. Por ejemplo: "Voy a ser el presidente de } \\
\text { la ciencia, la tecnología, la innovación, la protección de } \\
\text { los animales y la protección del medio ambiente" (Iván } \\
\text { Duque). }\end{array}$ \\
\hline & $\begin{array}{c}\text { Atacar al oponente } \\
(0-1)\end{array}$ & $\begin{array}{l}\text { Publicaciones en las que el candidato ataca a un } \\
\text { oponente. Por ejemplo: "Los ciudadanos estamos listos } \\
\text { para debatir con la corrupta politiquería representada } \\
\text { en Duque. Solo díganme día y hora iUn abrazo a toda } \\
\text { esa Colombia de cambio!" (Gustavo Petro). }\end{array}$ \\
\hline & $\begin{array}{c}\text { Defender al } \\
\text { candidato }(0-1)\end{array}$ & $\begin{array}{l}\text { Publicaciones en las que el candidato se defiende de una } \\
\text { acusación de sus oponentes. Por ejemplo: "Amiga } \\
\text { @Avrilizz esos camiones que muestras jamás fueron del } \\
\text { Distrito Capital ni lo son ahora. Son de un empresario } \\
\text { privado que los arrendó a la ciudad en la emergencia, } \\
\text { Una vez llegó la flota nueva propiedad del Distrito los } \\
\text { devolvimos" (Gustavo Petro). }\end{array}$ \\
\hline & $\begin{array}{c}\text { Comparar candidatos } \\
\qquad(0-1)\end{array}$ & $\begin{array}{l}\text { Publicaciones en las que el candidato se compara con } \\
\text { sus oponentes. Por ejemplo: "El voto del } 17 \text { de junio es } \\
\text { muy claro. O se vota por la corrupción, o se vota por la } \\
\text { decencia. O se vota por la guerra y la violencia, o se } \\
\text { vota por la Paz. O se vota por el agua y el páramo de } \\
\text { Santurbán" (Gustavo Petro). }\end{array}$ \\
\hline \multirow{3}{*}{$\begin{array}{l}\text { Enfoque del } \\
\text { mensaje }\end{array}$} & Personal (0-1) & $\begin{array}{l}\text { Publicaciones en las que el candidato hace referencia a } \\
\text { un tema personal. Por ejemplo: "Ésta es la mirada con } \\
\text { la que nos recibe nuestro gato Fígaro. Un amigo } \\
\text { consentido que ha unido a nuestra familia" (Iván } \\
\text { Duque). }\end{array}$ \\
\hline & Político (0-1) & $\begin{array}{l}\text { Publicaciones en las que el candidato hace referencia a } \\
\text { temas de la agenda política. Por ejemplo: "No voy a } \\
\text { aumentar la edad de pensión. No voy a quitar la pensión } \\
\text { que se hereda. No voy a acabar con Colpensiones, voy } \\
\text { a hacer una reforma de equidad para que los subsidios } \\
\text { de las pensiones no se queden en los ricos. } \\
\text { \#elfuturoesdetodos" (Iván Duque). }\end{array}$ \\
\hline & Promocional (0-1) & $\begin{array}{l}\text { Publicaciones netamente promocionales, como jingles } \\
\text { de campaña e imágenes del tarjetón electoral, invitando } \\
\text { a votar por el candidato. }\end{array}$ \\
\hline
\end{tabular}

Fuente: Elaboración propia, con base en la teoría del análisis funcional.

En la Figura 1, se aprecia el modelo de investigación para este artículo: 
Figura 1

Modelo de investigación

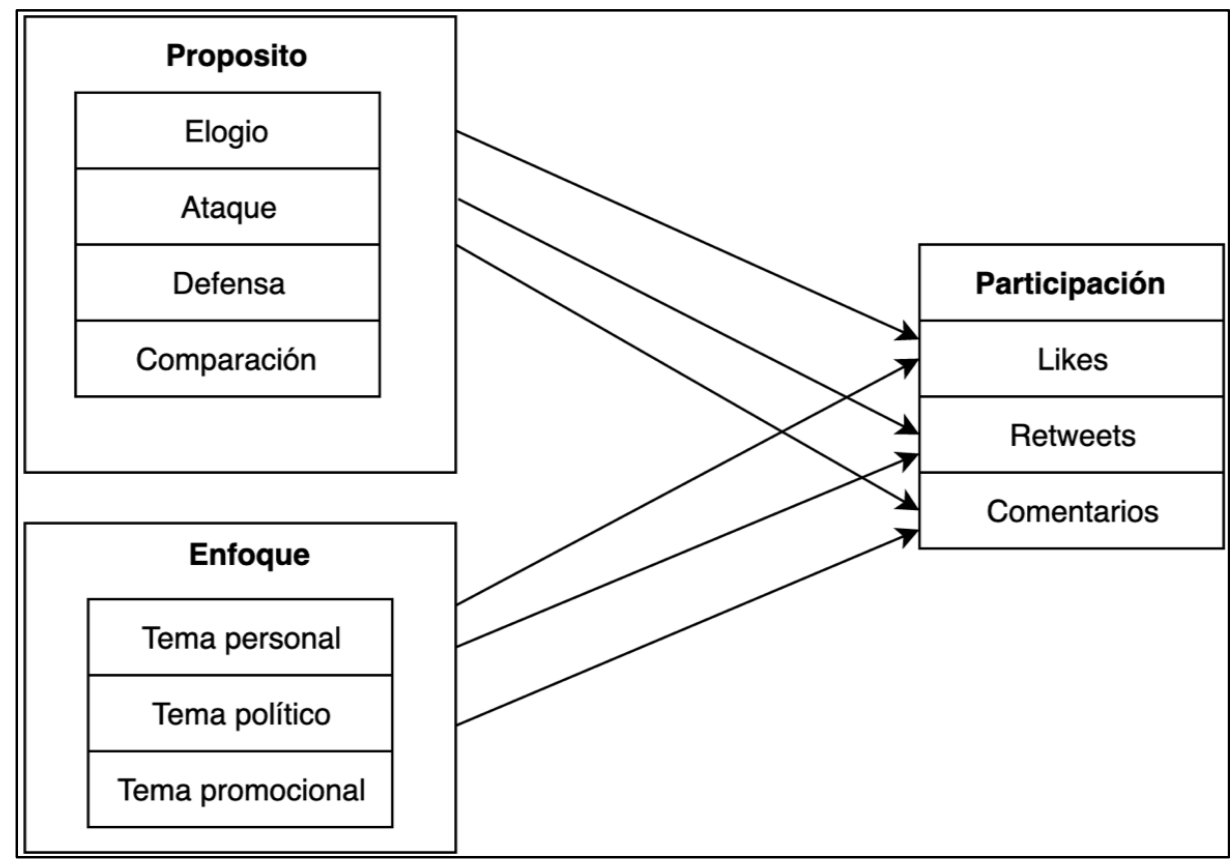

Fuente: Elaboración propia con base en la teoría del análisis funcional.

Fiabilidad de la codificación de las variables

Para garantizar la fiabilidad de la operacionalización de las publicaciones, se entrenó a dos personas para codificar. En las sesiones de capacitación, se incluyó la explicación de cada variable y sus categorías, a fin de que los codificadores entendieran las definiciones teóricas y operativas. Antes de la codificación real, se realizó una prueba piloto con 50 posts de twitter que no formaban parte de la muestra final. Se emplearon varias sesiones de entrenamiento y se modificó la plantilla de códigos hasta alcanzar la fiabilidad aceptable entre los codificadores. Para la muestra real, se utilizaron 100 tweets (30\% del total), que los codificadores analizaron para una verificación de fiabilidad. Las puntuaciones de fiabilidad de todas las variables fueron pertinentes, ya que estaban por encima de 0,80 en el alfa de Krippendorff (Krippendorff, 2018).

\section{Análisis empírico}

A continuación, se describe la metodología empleada para dar respuesta a las preguntas formuladas. Antes de empezar, se comprobó que las variables independientes no presentaran problemas de colinealidad perfecta. Para esto, se aplicó un modelo de 
regresión por mínimos cuadrados ordinarios (MCO) y se probó el factor de inflación de varianza (FIV). Todas las variables obtuvieron valores aceptables para el FIV, entre 0.02 y 4, lo cual prueba el supuesto de multicolinealidad (Hair et al., 2009). Como las variables dependientes escogidas para este artículo (likes, retweets y comentarios) fueron de recuento, es decir, provienen de un conteo, las técnicas tradicionales de regresión lineal por MCO no aplican para este caso. Particularmente, porque el supuesto de normalidad de los errores se viola, dado que los datos presentan asimetría positiva (Abitbol y Lee, 2017).

Para modelar conjuntos de datos discretos, una buena opción consiste en aplicar un modelo de regresión lineal generalizado (GML), el cual trata con la naturaleza discreta de las variables dependientes: las relaciona con las variables independientes, a través de una función de enlace, o "link function" (Cameron y Trivedi, 2013). Generalmente, los datos que proceden de un conteo siguen una distribución de Poisson; por tanto, se aplicó este modelo de regresión, para establecer los efectos de las variables independientes (propósito y enfoque del mensaje), sobre la participación de los usuarios en Twitter (Coxe, West y Aiken, 2009).

Una de las principales preocupaciones a la hora de aplicar la regresión de Poisson es que sus supuestos pueden ser poco realistas. Por ejemplo, el supuesto de que la media condicional y la varianza condicional de los datos deben ser iguales es algo que rara vez se cumple en la realidad. Y la sobredispersión puede conducir a la mala interpretación de los errores de los coeficientes, lo que deriva en la significancia estadística de factores que realmente no aportan al modelo (Navarro et al., 2001). Para lidiar con este problema, una buena alternativa es la regresión binomial negativa, la cual capta parte de la varianza que no se identifica en Poisson; así, se ajusta mejor al conjunto de datos (Ver Hoef y Boveng, 2007; Becerra y Vela, 2011). Dado lo anterior, se aplicó un modelo de regresión binomial negativa, como se evidencia en la ecuación 1 :

(1) $\ln (y)=\alpha+\beta_{1}$ acla $+\beta_{2}$ ata $+\beta_{3}$ def $+\beta_{4}$ comp $+\beta_{5}$ pers $+\beta_{6}$ poli $+\beta_{7}$ prom

Donde Ln (y) corresponde al logaritmo natural de la variable dependiente, la cual puede asumir una de tres formas: likes, retweets y comentarios; $\alpha$ es el término constante; acla significa aclamar; ata, atacar; def, defender y comp, comparar. Todas corresponden a las categorías de la variable propósito del mensaje, y presentan una naturaleza dicotómica; se operacionalizaron con uno (1), cuando estaban presentes y (0), cuando estaban ausentes. Por otro lado, las variables pers (personal), poli (política) y prom (promocional) hacen referencia a las categorías de la variable independiente del enfoque del mensaje. Estas variables también son de naturaleza discreta y se operacionalizaron con uno (1), cuando estaban presentes, y cero (0), cuando estaban ausentes. 


\section{Resultados}

Las estadísticas descriptivas se presentan en la Tabla 2. Se analizaron un total de 425 posts en Twitter de los dos candidatos a la segunda vuelta presidencial de Colombia, durante 2018. Los posts corresponden al periodo de tiempo del 27 de mayo al 17 de junio del mismo año. En promedio, se analizaron 212 posts por candidato. Entre los posts de Twitter, la cantidad de comentarios promedio recibidos fue de 186; la de likes de 2.998 y la de retweets de 1.191 . En relación con las categorías propuestas, para la variable propósito del mensaje, un $74 \%$ de las publicaciones utilizaron contenido, en el que el candidato se elogia a sí mismo; un $18 \%$ de las piezas eran de ataque hacia el oponente; el $8 \%$ eran publicaciones defendiéndose del ataque de otro candidato y un $4 \%$ fueron de comparación. Para la variable enfoque del mensaje, un $55 \%$ de las publicaciones se centraron en temas personales del candidato, como su familia, su mascota, su religión y sus pasatiempos. Un $52 \%$ se enfocó en promesas políticas o temas relacionados con la actualidad nacional y un $3 \%$ representa las piezas netamente promocionales.

Tabla 2

Estadísticos descriptivos

\begin{tabular}{|c|c|c|c|c|c|}
\hline \multicolumn{2}{|c|}{ Participación digital del elector } & Media & SD & Mínimo & Máximo \\
\hline \multicolumn{2}{|c|}{ Comentarios } & 186,78 & 279,81 & 14 & 2.700 \\
\hline \multicolumn{2}{|c|}{ Likes } & $2.998,48$ & 4400 & 49 & 44.500 \\
\hline \multicolumn{2}{|l|}{ Retweets } & 1190,99 & 1909,69 & 36 & 18.000 \\
\hline \multicolumn{6}{|c|}{ Variables categóricas } \\
\hline \multirow{5}{*}{$\begin{array}{l}\text { Propósito } \\
\text { del } \\
\text { mensaje }\end{array}$} & & \multicolumn{2}{|l|}{ Frecuencia } & \multicolumn{2}{|c|}{ Frecuencia relativa $(\%)$} \\
\hline & Aclamar & \multicolumn{2}{|l|}{317} & \multicolumn{2}{|c|}{76,61} \\
\hline & Ataque & \multicolumn{2}{|l|}{75} & \multicolumn{2}{|c|}{17,69} \\
\hline & Defensa & \multirow{2}{*}{\multicolumn{2}{|c|}{$\frac{35}{17}$}} & \multirow{2}{*}{\multicolumn{2}{|c|}{$\begin{array}{l}8,25 \\
4,01\end{array}$}} \\
\hline & Comparación & 17 & & & \\
\hline \multirow{3}{*}{$\begin{array}{l}\text { Enfoque } \\
\text { del } \\
\text { mensaje }\end{array}$} & $\begin{array}{l}\text { Temas } \\
\text { personales }\end{array}$ & \multicolumn{2}{|l|}{223} & \multicolumn{2}{|c|}{54,95} \\
\hline & Temas políticos & \multicolumn{2}{|l|}{220} & \multicolumn{2}{|c|}{51,89} \\
\hline & Promocic & \multicolumn{2}{|l|}{$\frac{\angle 2 U}{14}$} & & \\
\hline
\end{tabular}

Fuente: Elaboración propia.

A continuación, en la Tabla 3, se resumen los resultados de los modelos aplicados (likes, retweets y comentarios), para contrastar las hipótesis propuestas: 
Tabla 3

Modelo empírico

\begin{tabular}{|c|c|c|c|c|c|c|c|}
\hline \multirow{2}{*}{\multicolumn{2}{|c|}{ Variables dependientes }} & \multicolumn{2}{|c|}{ Likes } & \multicolumn{2}{|c|}{ Retweets } & \multicolumn{2}{|c|}{ Comentarios } \\
\hline & & $\beta$ & IRR & $\boldsymbol{\beta}$ & IRR & $\beta$ & IRR \\
\hline \multirow{4}{*}{$\begin{array}{l}\text { Propósito } \\
\text { del mensaje }\end{array}$} & Aclamar & $-0,786$ & 0,924 & $-0,155$ & 0,856 & $-0,440 * * *$ & $0,643 * * *$ \\
\hline & Atacar & $0,638 * * *$ & $1,893 * * *$ & $0,775 * * *$ & $2,170 * * *$ & $0,478 * * *$ & $1,613 * * *$ \\
\hline & Defender & $0,426 * *$ & $1,531 * *$ & $0,450 * *$ & $1,569 * *$ & $0,420 * *$ & $1,522 * *$ \\
\hline & Comparar & 0,316 & 1,371 & 0,237 & 1,267 & 0,302 & 1,352 \\
\hline \multirow{3}{*}{$\begin{array}{l}\text { Enfoque del } \\
\text { mensaje }\end{array}$} & Personales & $0,616 * * *$ & $1,853 * * *$ & $0,565 * * *$ & $1,760 * * *$ & $0,568 * * *$ & $1,766^{* * *}$ \\
\hline & Políticos & $-0,063$ & 0,938 & $-0,071$ & 0,930 & 0,046 & 1,004 \\
\hline & Promoción & $-0,670$ & 0,935 & $-0,718$ & 0,939 & 0,093 & 1,098 \\
\hline \multicolumn{2}{|l|}{$\operatorname{LR} X^{2}(7)$} & \multicolumn{2}{|c|}{98,61} & \multicolumn{2}{|c|}{131,43} & \multicolumn{2}{|c|}{132,16} \\
\hline \multicolumn{2}{|l|}{ Prob $>X^{2}$} & \multicolumn{2}{|c|}{0,0000} & \multicolumn{2}{|c|}{0,0000} & \multicolumn{2}{|c|}{0,0000} \\
\hline \multicolumn{2}{|c|}{ LR test de Alpha } & \multicolumn{2}{|c|}{$1,3 e+06$} & \multicolumn{2}{|c|}{$5,1 e+05$} & \multicolumn{2}{|c|}{$7,2 e+04$} \\
\hline \multicolumn{2}{|c|}{ Prob $>X^{2}$ Alpha } & \multicolumn{2}{|c|}{0,0000} & \multicolumn{2}{|c|}{0,0000} & \multicolumn{2}{|c|}{0,0000} \\
\hline
\end{tabular}

Fuente: Elaboración propia con base en la codificación.

*** $\mathrm{p}<0.01,{ }^{* *} \mathrm{p}<0.05,{ }^{*} \mathrm{p}<0.1$, IRR $=$ tasa de incidencia, LR test de Aplha = comparación entre modelos Binomial y Poisson.

Lo primero que se puede apreciar, del análisis empírico, es que los modelos son conjuntamente significativos, dado que el estadístico LR (Likelihood Ratio), o razón de verosimilitud que sigue una distribución de $X^{2}$, con 70 grados de libertad, arroja una probabilidad, en likes, de 98,61 > 0,0000; en retweets, de 131,43>0,0000, y en comentarios, de $132,16>0,0000$. Además, la prueba que existe sobre dispersión corregida, por parte del modelo binomial negativo, se refleja en el parámetro Alpha del modelo binomial: arroja una probabilidad, en likes, de 1,3e+06 > 0,0000; en retweets, de $5,1 e+05>0,0000$ y en comentarios, de 7,2e+04 > 0,0000. Estos parámetros indican que el modelo binomial negativo, efectivamente, está realizando una corrección en la sobredispersión de los datos. Por tanto, se ajusta mejor que el modelo de Poisson.

En relación con las variables likes y retweets, puede observarse que las piezas de ataque a un oponente político son altamente significativas: un nivel de confianza del $99 \%$ y un signo positivo de $\beta=0,638 * * *$. En otras palabras, ante el uso de piezas de ataque, la tasa de likes aumenta en 1,8 (IRR) veces. De manera similar, las piezas en las que los candidatos se defienden de ataques de otro candidato también presentan efectos significativos frente al número de likes, con un nivel de confianza del $95 \%$ y un signo positivo $\beta=0,426^{* *}$. Esto indica que, cuando se usan piezas en las que los candidatos se defienden de acusaciones o ataques de oponentes, la tasa de likes aumenta en 1,5 (IRR) veces. Las piezas de aclamación, comparación y promoción no presentan efectos significativos para likes.

En el modelo de la variable dependiente retweets, sucede algo parecido que en la variable anterior. Las piezas de ataque son altamente significativas: $99 \%$, y presentan un signo positivo de $\beta=0,775^{* * *}$. Esto quiere decir que, ante el uso de publicaciones de ataque, la tasa de retweets aumenta en 2,1 (IRR) veces. De manera similar, las piezas de 
defensa son significativas: $95 \%$, y presentan betas positivos de $\beta=0,450 * *$. En otras palabras, ante el uso de piezas de defensa, los retweets aumentan 1,5 (IRR) veces. Frente al enfoque del mensaje, las piezas de comunicación que tratan temas personales de los candidatos son altamente significativas: $99 \%$ de confianza, con un signo positivo de $\beta=$ $1,760 * * *$. Esto significa que, ante el uso de estas piezas, la tasa de retweets aumenta 1,7 (IRR) veces. Nuevamente, las categorías de aclamación, comparación, temas políticos y promoción no ejercen efectos significativos sobre la tasa de retweets.

Finalmente, en la variable comentarios, se puede apreciar que las publicaciones de ataque generan efectos altamente significativos, con un nivel de confianza del $99 \%$ y coeficientes positivos ( $\beta=0,478 * * *$ ). Dicho de otra manera, al utilizar piezas de ataque, la tasa de comentarios aumenta 1,6 (IRR) veces. Las piezas defensivas también son significativas, esta vez con un $95 \%$ de confianza y un signo positivo de $\beta=0,420 * *$. Lo cual indica que, ante el uso de este tipo de publicaciones, la tasa de comentarios aumenta 1,5 (IRR) veces. Para este modelo, las piezas de aclamación propias del candidato son altamente significativas, con un nivel de confianza del $99 \%$ y un signo negativo que presenta un $\beta=-0,440 * * *$. Estos resultados sugieren que, ante el uso de piezas de aclamación, la tasa de comentarios será 0,6 (IRR) veces menor. En relación con el enfoque del mensaje, se puede observar que las publicaciones en las que se comparte contenido personal de los candidatos son altamente significativas, con un nivel de confianza del $99 \%$ y un signo positivo de $\beta=0,568 * * *$. Esto significa que el uso de estas publicaciones aumenta la tasa de comentarios en 1,7 (IRR) veces. Para este modelo, las categorías de piezas promocionales, políticas y comparativas no son estadísticamente significativas.

\section{Conclusiones}

A partir de este estudio, es posible, a modo de reflexión, concluir que:

Se identificaron las características más utilizadas de los mensajes políticos en la campaña a la presidencia de Colombia en 2018. Una de esas características es: la mayoría de las piezas de comunicación se orientan a temas personales del candidato; lo cual es fallido, porque, en este lugar, deberían estar aquellos mensajes enfocados en las propuestas, o los temas de interés político. En efecto, las publicaciones sobre temas personales de los candidatos, como sus emociones, su familia, sus pasatiempos e incluso sus mascotas reciben mayor respaldo social en Twitter, a través de likes, retweets y comentarios. En otras palabras, si los candidatos desean mejorar el respaldo social de sus usuarios de Twitter, deben utilizar, con mayor frecuencia, piezas en las que se toquen temas personales y emocionales. Pero no deberían comunicar sus propuestas en estos medios.

Otra de las características es que aquellas piezas de comunicación en las que un candidato ataca a sus oponentes gozan de mayor respaldo en Twitter. $Y$, aunque en menores proporciones, las publicaciones de contenido defensivo (cuando un candidato se 
defiende de un ataque de la oposición) aumentan la participación política de los ciudadanos en redes sociales; esto no sucede cuando los candidatos publican piezas positivas hablando bien de sí mismos. En otras palabras, los usuarios de redes sociales en Colombia se sienten más atraídos hacia campañas políticas que promueven el enfrentamiento y la división que las que proponen soluciones para los problemas del país. Este comportamiento de los usuarios en redes sociales frente a las piezas de publicidad política indica que Colombia se encuentra todavía en un estado de inmadurez democrática (Larreguy, Marshall y Snyder, 2018). Por este motivo, es necesario seguir explorando acerca de las motivaciones de los internautas colombianos frente a los procesos electorales.

Estos hallazgos contribuyen al clásico debate sobre la efectividad de la publicidad política negativa o agresiva. En general, los resultados de esta investigación sugieren que la propaganda negativa difundida en Twitter contradice, dentro del contexto de las redes sociales, los hallazgos seminales de Ansolabehere y Iyengar (1994), y los resultados anteriores de Pinkleton, Um y Austin (2002) y Shah et al (2007), quienes afirman que la publicidad negativa conduce a la "desmovilización" del electorado, es decir, a la disminución de la participación en procesos electorales. Por el contrario, el presente artículo respalda las ideas de Finkle y Geer (1998), Freedman y Goldstein (1999) y Brians y Wattenberg (1996), quienes defienden la hipótesis de estimulación, en otras palabras, sostienen que la publicidad negativa tiene un efecto estimulante en el electorado aumentando su participación de manera significativa: este fue el caso de Colombia, donde la participación electoral fue la mayor en los últimos veinte años (Igac, 2018).

En síntesis, este artículo coadyuva a la comprensión del papel que juega la publicidad política en la participación de los ciudadanos a través de medios sociales. De hecho, abre la posibilidad de que futuras investigaciones efectúen un análisis a una escala mayor; por ejemplo, tomando periodos de tiempo más largos para la recolección de datos, o estableciendo contrastes entre países. Realmente, sería interesante comparar los resultados de este artículo con otros que se realicen en países cuyas democracias sean maduras y establecidas. También sería interesante adelantar modelos similares en otras redes sociales; es bien sabido que el público objetivo de cada red social puede diferir en su comportamiento, por lo tanto, los hallazgos de esta investigación pueden compararse con otros medios sociales como Facebook o Instagram, para analizar la respuesta de los internautas ante la exposición a diferentes tipos de publicidad política.

\section{Referencias}

ABBE, O., et al. "Agenda setting in congressional elections: the impact of issues and campaigns on voting behavior". Political Research Quarterly, vol. 56, no 4, p. 419-430, dic. 2003.

Abitbol, A.; LEE, S. "Messages on CSR-dedicated Facebook pages: what works and what doesn't". Public Relations Review, vol. 43, no 4, p. 796-808, nov. 2017. 
AnsolABeHeRE, S.; IYengaR, S. "Riding the wave and claiming ownership over issues: the joint effects of advertising and news coverage in campaigns". Public Opinion Quarterly, vol. 58, no 3, p. 335-357, 1994.

AnsolAbehere, S., et al. "Does attack advertising demobilize the electorate?". American Political Science Review, vol. 88, no 4, p. 829-838, 1994.

BASIL, M.; SCHOOler, C.; Reeves, B. Positive and negative political advertising: effectiveness of ads and perceptions of candidates. In: BIoccA, F. (ed.). Television and political advertising. Vol. 1, Psychological processes; Vol. 2, Signs, codes, and images (p. 245-262). New Jersey: Lawrence Erlbaum Associates, Inc. 1991.

BeCERRA, N.; VelA, F. "Una comparación de los modelos Poisson y binomial negativa con Stata: un ejercicio didáctico". In: 3er Encuentro de Usuarios de Stata. México, D.F., p. 1-23, mayo 2011.

BENOIT, W. "A functional analysis of political advertising across media, 1998". Communication Studies, vol. 51, no 3, p. 274-295, 2000.

. "The functional approach to presidential television spots: acclaiming, attacking, defending 1952-2000". Communication Studies, vol. 52, no 2, p. 109-126, 2001.

BENOIT, W.; KLYUKovSKI, A. "A functional analysis of the 2004 Ukrainian debates". Argumentation, vol. 20, no 2, p. 209-225, sep. 2006.

BenOIT, W.; SheAfER, T. "Functional theory and political discourse: televised debates in Israel and the United States". Journalism \& Mass Communication Quarterly, vol. 83, no 2, p. 281-297, jun. 2006.

Benoit, W.; Wen, W.; YU, T. "A functional analysis of 2004 Taiwanese political debates". Asian Journal of Communication, vol. 17, no 1, p. 24-39, mar. 2007.

Berelson, B.; LazARsfeld, P.; McPhee, W. Voting: a study of opinion formation in a presidential campaign. University of Chicago Press: Midway Reprint, 1954.

BORAH, P. "Political Facebook use: campaign strategies used in 2008 and 2012 presidential elections". Journal of Information Technology and Politics, vol. 13, no 4, p. 326-338, mayo 2016.

BorBA, F. "Propaganda negativa nas eleições presidenciais de 2010: o caminho para José Serra?". Em debate: Periódico de Opinião Pública e Conjuntura Política, ano 2, no 8, ago. 2010.

BRADER, T. "Striking a responsive chord: how political ads motivate and persuade voters by appealing to emotions". American Journal of Political Science, vol. 49, no 2, p. 388-405, abr. 2005.

BRIANS, C. L.; WATtenBerg, M. P. "Campaign issue knowledge and salience: comparing reception from TV commercials, TV news and newspapers". American Journal of Political Science, p. 172-193, 1996.

Cameron, C.; TRIVedi, P. Regression analysis of count data. $2^{\text {nd }}$ ed. (en línea). Cambridge: Cambridge University Press. doi:10.1017/CBO9781139013567, 2013.

Chaffee, S.; ChOE, S. "Time of decision and media use during the Ford-Carter campaign". Public Opinion Quarterly, vol. 44, no 1, p. 53-69, ene. 1980.

COXE, S.; WeSt, S.; AIKEN, L. "The analysis of count data: a gentle introduction to Poisson regression and its alternatives". Journal of Personality Assessment, vol. 91, no 2, p. 121-136, feb. 2009.

DALTON, R. The good citizen: how a younger generation is reshaping American politics. $2^{\text {a }}$ ed. New York: CQ Press, 2015. 
De VRIeS, L.; Gensler, S.; Leeflang, P. "Popularity of brand posts on brand fan pages: an investigation of the effects of social media marketing". Journal of Interactive Marketing, vol. 26, $\mathrm{n}^{\circ}$ 2, p. 83-91, mayo 2012.

DimmiCK, J.; CHEN, Y.; LI, Z. "Competition between the internet and traditional news media: the gratification-opportunities niche dimension". Journal of Media Economics, vol. 17, no 1, p. 19-33, 2004.

EvarTS, D.; GuIDO, H. "Coverage of the 1972 campaign by TV, news magazines and major newspapers". Journalism Quarterly, vol. 51, no 4, p. 645-648, dic. 1974.

FinKLE, S. E.; GeER, J. "A spot check: casting doubt on the demobilizing effect of attack advertising". American Journal of Political Science, vol. 42, no 2, p. 573-595, 1998.

FLEW, T. New media: an introduction. $3^{\text {a }}$ ed. Oxford: Oxford University Press, 2007.

Franz, M.; Ridout, T. "Does political advertising persuade?". Political Behavior, vol. 29, n 4, p. 465491, dic. 2007.

Freedman, P.; GoldSTEIN, K. "Measuring media exposure and the effects of negative campaign ads". American Journal of Political Science, p. 1189-1208, 1999.

GARRAMONE, G. M. "Effects of negative political advertising: the roles of sponsor and rebuttal". Journal of Broadcasting \& Electronic Media, vol. 29, no 2, p. 147-159, 1985.

Goldstein, K.; Freedman, P. "Campaign advertising and voter turnout: new evidence for a stimulation effect". The Journal of Politics, vol. 64, no 3, p. 721-740, 2002.

HAIR, J., et al. Multivariate data analysis. London: Prentice Hall, 2009.

HARRIS, R.; SANBORn, F. A cognitive psychology of mass communication. New York: Taylor \& Francis Group, 2009.

Herrero, J.; Benoit, W. "Análisis funcional de los debates en las elecciones generales de 2008". ZER: Revista de Estudios de Comunicación, vol. 14, no 27, p. 61-81, sep. 2009.

IgAC - Instituto Geográfico Agustín CodAzZi. Mapeando la abstención electoral de Colombia en el siglo XXI, 2018. Disponible en: <https://igac.gov.co/es/noticias/mapeando-la-abstencion-electoralde-colombia-en-el-siglo-xxi/>. Accesado el: 20 mayo 2020.

KAID, L. "TechnoDistortions and effects of the 2000 political advertising". American Behavioral Scientist, vol. 44, no 12 , p. 2.370-2.378, ago. 2001.

KAID, L.; BACHA, C. Television advertising and democratic systems around the world: a comparison of videostyle content and effects. In: KAID, L.; BACHA, C. The Sage handbook and political advertising. Newbury Park, California: Sage Publications, p. 445-458, 2006.

KAID, L.; POSTELnICU, M. "Political advertising in the 2004 election: comparison of traditional television and internet messages". American Behavioral Scientist, vol. 49, nº 2, p. 265-278, oct. 2005.

KRIPPENDORFF, K. Content analysis: an introduction to its methodology. Los Ángeles: Sage Publications, 2018.

LARREguY, H.; MARShALL, J.; SNYDer, J. "Leveling the playing field: how campaign advertising can help non-dominant parties". Journal of the European Economic Association, vol. 16, no 6, p. 1.812-1.849, abr. 2018. 
PUBLICIDAD POLÍTICA Y SU INCIDENCIA EN LA PARTICIPACIÓN DE USUARIOS EN REDES SOCIALES

LARSON, L.; DENTON, T. "eWOM Watchdogs: ego-threatening product domains and the policing of positive online reviews". Psychology \& Marketing, vol. 31, no 9, p. 801-811, ago. 2014.

LEE, C.; Benoit, W. "A functional analysis of the 2002 Korean presidential debates". Asian Journal of Communication, vol. 15, no 2, p. 115-132, 2005.

MANGold, W.; FAULDS, D. "Social media: the new hybrid element of the promotion mix". Business Horizons, vol. 52, no 4, p. 357-365, jul.-ago. 2009.

MERRITT, S. "Negative political advertising: some empirical findings". Journal of Advertising, vol. 13, no 3, p. 27-38, 1984.

NAvarro, A., et al. "La distribución binomial negativa frente a la de Poisson en el análisis de fenómenos recurrentes". Gaceta Sanitaria, vol. 15, no 5, p. 447-452, 2001.

PATHAK, A.; KUMAR, S. "Independent regulation of tumor cell migration by matrix stiffness and confinement". Proceedings of the National Academy of Sciences, vol. 109, no 26, p. 10334-10339, 2012.

PinkLeton, B. E.; UM, N. H.; AUSTIN, E. W. "An exploration of the effects of negative political advertising on political decision making". Journal of Advertising, vol. 31, no 1, p. 13-25, 2002.

RAO, A.; LEE, A.; KIM, H. "It's time to vote: the effect of matching message orientation and temporal frame on political persuasion". Journal of Consumer Research, vol. 35, nº 6, p. 877-889, abr. 2009.

Ridout, T. N.; Franz, M. M.; FoWLER, E. F. "Sponsorship, disclosure, and donors: limiting the impact of outside group ads". Political Research Quarterly, vol. 68, no 1, 154-166, 2015.

RidouT, T., et al. "The long-term and geographically constrained effects of campaign advertising on political polarization and sorting". American Politics Research, vol. 46, no 1, p. 3-25, jul. 2017.

Seerat, S.; Hansandaldeep, K. "A content analysis of YouTube political advertisements: evidence from Indian parliamentary elections". Journal of Creative Communications, vol. 13, no 2, p. 133156, abr. 2018.

SHAH, D., et al. "Campaign ads, online messaging, and participation: extending the communication mediation model". Journal of Communication, vol. 57, no 4, p. 676-703, dic. 2007.

Sмiтh, A. Digital politics: Pew Research findings on technology and campaign. Washington: Pew Internet Researcher, 2013.

SOHAL, S.; KAUR, H. "A content analysis of YouTube political advertisements: evidence from Indian parliamentary elections". Journal of Creative Communications, vol. 13, no 2, p. 133-156, abr. 2018.

SOLEY, L.; REID, L. "Promotional expenditures in U.S. congressional elections". Journal of Marketing \& Public Policy, vol. 1, p. 147-155, 1982.

SPENKUCH, J.; TONIATTI, D. "Political advertising and election results". Quarterly Journal of Economics, vol. 133, no 4, p. 1.981-2.036, mayo 2018.

TAFESSE, W.; KoRneliUSSEN, T. "Examining the effect of using multiple media tools on the marketing performance of organizations in a trade campaign environment". Journal of Marketing Communications, vol. 19, no 3, p. 1-13, ene. 2012.

Valentino, N.; Hutchings, V.; Williams, D. "The impact of political advertising on knowledge, internet information seeking, and candidate preference". Journal of Communication, vol. 54, no 2, p. 337-354, jun. 2004. 
VAN SteenbuRG, E. "Areas of research in political advertising: a review and research agenda". International Journal of Advertising, vol. 34, n 2, p. 195-231, ene. 2015.

VER HOEF, J.; BOVEnG, P. "Quasi-Poisson vs. negative binomial regression: how should we model overdispersed count data?". Ecology, vol. 88, n 11, p. 2.766-2.772, nov. 2007.

WATtenberg, M. P.; BRIANS, C. L. "Negative campaign advertising: demobilizer or mobilizer?". American Political Science Review, vol. 93, n 4, p. 891-899, 1999.

Williams, C.; GULATI, G. "Digital advertising expenditures in the 2016 presidential election". Social Science Computer Review, vol. 36, no 4, p. 406-421, sep. 2017.

\begin{abstract}
Political advertising and its impact on the participation of users in social networks: presidential elections of Colombia 2018

The research presented in this article analyzes the strategies of the political announcements published on Twitter by the candidates for the second round of presidential election in Colombia, in the year 2018. The purpose and focus of the message of 425 posts was identified and a negative binomial regression model was applied to establish the effects of these elements on the participation of users on Twitter measured by likes, comments and retweets. There were two relevant findings: a) aggressive political advertising - when candidates attack, or defend against an opponent - increases the participation of users on Twitter; b) Messages with personal themes of the candidate have greater social support than publications that address political proposals.
\end{abstract}

Keywords: political advertising; Twitter; emotions

\title{
Resumo
}

Publicidade política e seu impacto na participação de usuários nas redes sociais: eleições presidenciais da Colômbia 2018

A pesquisa apresentada neste artigo analisa as estratégias dos anúncios políticos publicados no Twitter pelos candidatos para o segundo turno da eleição presidencial da Colômbia, em 2018. Foram identificados o propósito e o enfoque da mensagem de 425 posts e foi aplicado um modelo de regressão binomial negativo, para estabelecer os efeitos desses elementos na participação dos usuários no Twitter, medidos por número de curtidas, comentários e retweets. Houve duas descobertas relevantes: a) a publicidade política agressiva - quando os candidatos atacam ou se defendem contra um adversário - aumenta a participação do usuário no Twitter; b) as mensagens com questões pessoais do candidato têm maior apoio social do que publicações que abordam propostas políticas.

Palavras-chave: publicidade política; Twitter; emoções

\section{Résumé}

Publicité politique et son impact sur la participation des utilisateurs aux réseaux sociaux: élections présidentielles de Colombie 2018

La recherche présentée dans cet article analyse les stratégies des messages politiques publiées sur Twitter par les candidats au deuxième tour des élections présidentielles de Colombie en 2018 L'objectif et l'approche du message des 425 posts ont été identifiées. On a utilisé un modèle de régression binomiale négative pour établir les effets de ces éléments sur la participation des utilisateurs sur Twitter, étant mesurés par les likes, les commentaires et les retweets. On arrive à deux conclusions pertinentes: a) la publicité politique agressive - lorsque les candidats attaquent ou se défendent face à un adversaire - augmente la participation des utilisateurs sur Twitter; b) les 
messages concernant les problèmes personnels du candidat bénéficient d'un plus grand soutien social que les publications qui expriment leurs programmes politiques.

Mots-clés: publicité politique; Twitter; émotions

Artigo submetido à publicação em 24 de janeiro de 2020. Versão final aprovada em 8 de setembro de 2020. Opinião Pública adota a licença Creative Commons CC-BY. 